\title{
A NECESSARY AND SUFFICIENT CONDITION FOR $\beta X \backslash X$ TO BE AN INDECOMPOSABLE CONTINUUM
}

\author{
R. F. DICKMAN, JR.
}

\begin{abstract}
In his dissertation, David Bellamy has shown that if $I=[0,1)$, then $\beta I: I$ is an indecomposable continuum, and R. G. Woods, in his dissertation, obtained the same result and in addition showed that for $m>1, \beta R^{m} \backslash R^{m}$ is a decomposable continuum. In this note we give a necessary and sufficient condition for $\beta X \backslash X$ to be an indecomposable continuum when $X$ is a locally connected generalized continuum.
\end{abstract}

Definitions and notation. If $Y$ is a topological space and $A \subset B \subset Y$, we denote the closure of $A$ in $B$ by $\mathrm{cl}_{B} A$ and we say that a set $A \subset B$ is conditionally compact in $B$ if $\mathrm{cl}_{B} A$ is compact. A ray in $Y$ is a closed subset $R$ of $Y$ that is homeomorphic to $[0,1)$. We say that a connected space $Y$ has the strong complementation property provided whenever $U$ is a nonconditionally compact connected open subset of $Y, Y \backslash U$ is compact. A compact connected Hausdorff space $Y$ is said to be an indecomposable continuum if there does not exist two nonempty closed, connected proper subsets of $Y$ whose union is $Y$. By a generalized continuum we will mean a locally compact, connected metric space. We will use $\beta X$ to denote the Stone-Cech compactification of a completely regular Hausdorff space. All terms not defined here may be found in [6], [7] or [8].

We need the following results:

Lemma 1 [7, TheOREM 3.41]. Let $Y$ be a compact, connected Hausdorff space and $C$ be a nonempty, closed and connected proper subset of $Y$. If $C$ has a nonempty interior, $Y$ is not an indecomposable continuum.

LemMa 2. Let $X$ be a noncompact, locally connected generalized continuum. Then $X$ contains a ray.

Proof. Let $X_{\infty}$ denote the one-point compactification of $X$. It is well known that $X_{\infty}$ is a locally connected, compact connected metric space and as such is arcwise connected. Let $a \in X$ and let $I$ be any arc in $X_{\infty}$ from $a$ to $\{\infty\}$. Then $I \backslash\{\infty\}$ is a ray in $X$.

Received by the editors January 14, 1971.

AMS 1970 subject classifications. Primary 54D35; Secondary 54F15.

Key words and phrases. Stone-Cech compactification, indecomposable continuum.

(c) American Mathematical Society $19 / 2$ 
LEMMA 3. Let $X$ be a noncompact, locally connected generalized continuum. Then $X$ has the strong complementation property if and only if for every ray $R$ in $X, X \backslash R$ is conditionally compact.

Proof of THE NeCESSITy. Let $R$ be a ray in $X$ and suppose that $X \backslash R$ is not conditionally compact. Then there exists a closed set $A$ of $X$ such that $A$ is not compact and $A \cap R=\varnothing$. Since $X$ is locally connected there exists an open connected set $U$ of $X$ containing $R$ such that $A \cap U=\varnothing$. But then neither $\mathrm{cl}_{\mathrm{X}} U$ or $X \backslash U$ is compact and so $X$ cannot have the strong complementation property. This completes the proof of the necessity.

Proof of the sufficiency. Suppose that for every ray $R$ in $X, X \backslash R$ is conditionally compact. Let $U$ be any nonconditionally compact open "connected subset of $X$. By Lemma 2, $X$ contains a ray and so there exists a homeomorphism of $h$ of $[0,1)$ onto a closed set $S$ of $X$. Since $\operatorname{cl}_{X}(X \backslash S)$ is compact there exists a point $t \in[0,1)$ such that $h(t)$ is the last point on $S$ that lies in $\mathrm{cl}_{X}(X \backslash S)$. Then $h(t, 1)$ is an open subset of $X$. We assert that there exists $s \in[t, 1)$ such that $h[s, 1) \subset U$. First we note that for every $s \in[t, 1), h[s, 1) \cap U \neq \varnothing$ for otherwise, since $X \backslash h[s, 1)$ is conditionally compact, $U$ would be conditionally compact. Now suppose that for every $s \in[t, 1), h[s, 1)$ is not a subset of $U$. Then there exists $p \in(t, 1)$ such that $h(p) \notin U$ and both of the sets $h[t, p)$ and $h(p, 1)$ intersect $U$. Now $U_{1}=$ $U \cap(X, h[p, 1))$ is open in $X$ since $h[p, 1)$ is closed and $U_{2}=U \cap h(p, 1)$ is open in $X$ since $h(p, 1)$ is open in $X$. Furthermore, neither $U_{1}$ or $U_{2}$ is empty and $U=U_{1} \cup U_{2}$. Of course, this contradicts the assumption that $U$ is connected and so there exists $s \in[t, 1)$ such that $h[s, 1) \subset U$. Then $X \backslash U$ is a subset of $X \backslash h[s, 1)$ and so $X \backslash U$ is compact. This completes the proof.

THEOREM. Let $X$ be a noncompact locally connected generalized continuum. Then a necessary and sufficient condition for $\beta X \backslash X$ to be an indecomposable continuum is that $X$ have the strong complementation property.

Proof of the sufficiency. Suppose that $X$ has the strong complementation property. Let $R$ be any ray in $X$ and let $T=\operatorname{cl}_{\beta \mathrm{X}} R$. By D. Bellamy's result, $\beta R \backslash R$ is an indecomposable continuum and by Theorem $7 \mathrm{cf}$ [4], the identity mapping on $R$ can be extended to a homeomorphism of $T$ onto $\beta R$. Thus, $C=T R$ is an indecomposable continuum that lies in $\beta X \backslash X$. But by Lemma 3, $\operatorname{cl}_{X}(X \backslash R)$ is compact so that $\beta X \backslash X=C$ and $\beta X ! X$ is an indecomposable continuum.

Proof of the NeCESSITY. Suppose that $X$ does not have the strong complementation property. By Lemma 2, there exists a ray $R$ in $X$ such that $\mathrm{cl}_{X^{\prime}}\left(X^{\backslash} R\right)$ is not compact. Then there exists a noncompact closed subset $A$ of $X$ such that $A \cap R=\_$and a connected open subset $U$ of $X$ such that $R \subset U$ and $\mathrm{cl}_{X} U \cap A=\odot$. Now since $X$ is locally compact and 
separable $X=\bigcup K_{i}, i=1,2, \cdots$, where for each $i \geqq 1, K_{i}$ is a compact subset of the interior of $K_{i+1}$. Let $R_{1}, R_{2}, \cdots$ be a sequence of subrays of $R$ such that for each $i \geqq 1, R_{i}$ is a subset of $X \backslash K_{i}$. Finally for each $i \geqq 1$ let $G_{i}$ be the component of $U \cap\left(X \backslash K_{i}\right)$ that contains $R_{i}$ and let $H_{i}=\left(X \backslash K_{i}\right) \cap$ $\left(X \backslash G_{i}\right)$. We set $G=\cap \operatorname{cl}_{\beta X} G_{i}, i=1,2, \cdots$, and $H=\bigcap \operatorname{cl}_{\beta X} H_{i}, i=1,2, \cdots$, and note that $\beta X \backslash X=H \cup G$. Furthermore, we observe that $G$ is a nonempty compact, connected space. Now if $\beta X \backslash X$ is not connected, it cannot be an indecomposable continuum so suppose that $\beta X \backslash X$ is connected. We now proceed to show that $G$ is a proper subset of $\beta X \backslash X$ and that $G$ has a nonempty interior. Let $a \in \mathrm{cl}_{\beta X^{\mathrm{S}}} A \cap(\beta X \backslash X)$. Note that such a point exists since $A$ is not compact. Then since $X$ is a normal space, $A$ and $\mathrm{cl}_{X} U$ are completely separated in $X$ and by Theorem (6.5) of [6], $A$ and $\operatorname{cl}_{X} U$ have disjoint closures in $\beta X$. Then $a \notin \mathrm{cl}_{\beta X} U \supset G$ and so $G \neq \beta X \backslash X$. Similarly if $r \in \mathrm{cl}_{\beta X} R_{1} \cap(\beta X \backslash X), r \notin \mathrm{cl}_{\beta X} H_{1} \supset H$ and so $G$ has a nonempty interior relative to $\beta X \backslash X$. By Lemma $1, \beta X \backslash X$ is not an indecomposable continuum and this completes the proof.

COROllary. Let $X$ be a noncompact locally connected generalized continuum. If $\beta X \backslash X$ is an indecomposable continuum, then $X_{\infty}$, the one-point compactification of $X$, is the only locally connected compactification of $X$.

Proof. Suppose that $Y$ is a locally connected compactification of $X$ and $Y \backslash X$ is nondegenerate. Then there exists disjoint arcs $I_{1}$ and $I_{2}$ in $Y$ such that $I_{i} \cap(Y \backslash X)$ is a single point $a_{i}, i=1,2$. Then if $R_{i}=I_{i} \backslash\left\{a_{i}\right\}, i=1$, 2, $R_{1}$ is a ray in $X$ with $X \backslash R_{1}$ nonconditionally compact. By Lemma $3, X$ does not have the strong complementation property and hence $\beta X \backslash X$ cannot be an indecomposable continuum. Of course, this implies that $X_{\infty}$ is the only locally connected compactification of $X$.

EXAMPLE. Let $T_{0}=[0,1)$ and for each positive integer $n$ let $T_{n}$ be the closed line segment joining the points $(n-1 / n, 1)$ and $(n-1 / n, 0)$ in $E^{2}$. Let $X=\bigcup T_{i}, i=0,1,2, \cdots$. Then $X$ is a locally connected generalized continuum that does not have the strong complementation and so $\beta X \backslash X$ is not indecomposable. However, $X_{\infty}$ is the only locally connected compactification of $\bar{X}$.

REMARK. A connected space $Y$ is said to have the complementation property if for every compact set $K$ in $Y, Y \backslash K$ has at most one nonconditionally compact component It can be shown that if $X$ is a locally connected generalized continuum, then $X$ has the complementation if and only if $\beta X \backslash X$ is connected. Then by the theorem above, $X$ has the complementation property whenever it has the strong complementation property. See [2] or [3] for further properties of spaces with the complementation property. 


\section{REFERENCES}

1. David Bellamy, Topological properties of compactifications of a half-open interval, Ph.D. Thesis, Michigan State University, East Lansing, Mich., 1968.

2. R. F. Dickman, Jr., Unicoherence and related properties, Duke Math. J. 34 (1967), 343-351. MR 35 \#A3632.

3. - Compactness of mappings on products of locally connected generalized continua, Proc. Amer. Math. Soc. 18 (1967), 1093-1094. MR 36 \#853.

4. M. H. Stone, On the compactification of topological spaces, Ann. Soc. Polon. Math. 21 (1948), 153-160. MR 10, 137.

5. R. G. Woods, Certain properties of $\beta X: X$ for $\sigma$-compact $X$, Ph.D. Thesis, McGill University, 1968.

6. L. Gillman and M. Jerison, Rings of continuous functions, The University Series in Higher Math., Van Nostrand, Princeton, N.J., 1960. MR 22 \#6994.

7. J. G. Hocking and G. S. Young, Topology, Addison-Wesley, Reading, Mass., 1961. MR 23 \#A2857.

8. G. T. Whyburn, Analytic iopology, Amer. Math. Soc. Colloq. Publ., vol. 28, Amer. Math. Soc., Providence, R.I., 1942. MR 4, 86.

Department of Mathematics, University of Miami, Coral Gables, Florida 33124

Department of Mathematics, Virginia Polytechnic Institute and State UniVERSITY, BL.ACKSBURG, VIRGINIA 24061 